\title{
CHSY1 promoted proliferation and suppressed apoptosis in colorectal cancer through regulation of the NFKB and/or caspase-3/7 signaling pathway
}

\author{
LIFENG ZENG $^{1}$, JINRONG QIAN ${ }^{2}$, XIAOJIANG LUO $^{3}$, AIQUN ZHOU ${ }^{1}$, \\ ZHIYONG ZHANG ${ }^{1}$ and QUANGANG FANG ${ }^{1}$ \\ Departments of ${ }^{1}$ Clinical Laboratory, ${ }^{2}$ Health Care of Cadre and ${ }^{3}$ Gastrointestinal Surgery, \\ Jiangxi Province People's Hospital, Nanchang, Jiangxi 330006, P.R. China
}

Received December 31, 2017; Accepted July 30, 2018

DOI: $10.3892 / \mathrm{ol} .2018 .9385$

\begin{abstract}
Colorectal cancer is a commonly observed malignant cancer. However, the limited therapies for colorectal cancer do not bring much benefit for patients. Chondroitin synthase-1 (CHSY1) is an enzyme responsible for the biosynthesis of chondroitin sulfate and has been implicated in the tumorigenesis of several cancer types; however, there is limited information regarding the role of CHSY1 in colorectal cancer. In the present study, CHSY1 was demonstrated to be highly expressed in colorectal cancer tissues and in cell lines, and the CHSY1 expression level was associated with the 5-year survival rate of patients with colorectal cancer. Following CHSY1 knockdown, the proliferation of colorectal cancer cells was significantly decreased. The number of RKO cells decreased by $50 \%$ following CHSY1 knockdown compared with that in the control after culture for 5 days. However, the apoptosis rate of RKO cells increased to $14.15 \%$ after CHSY1 knockdown. In addition, the activity of caspase-3/7 was also enhanced. Furthermore, the expression of B-cell lymphoma 2 (Bcl-2) was reduced, whereas the levels of Bcl-2-associated $\mathrm{X}$ protein (Bax) and truncated caspase-3/7 were increased following CHSY1 knockdown. Additionally, the phosphorylation level of IкB and the expression of nuclear factor (NF) $\kappa \mathrm{B}$ also decreased. In contrast, forced expression of CHSY1

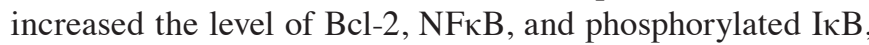
whereas the level of bax and truncated caspase-3/7 decreased. Therefore, the data of the present study suggest that CHSY1 promoted cell proliferation by regulating $\mathrm{NF \kappa B}$ signaling and suppressed cell apoptosis by regulating/caspase-3/7 signaling
\end{abstract}

Correspondence to: Dr Lifeng Zeng, Department of Clinical Laboratory, Jiangxi Province People's Hospital, 92 Aiguo Street, Nanchang, Jiangxi 330006, P.R. China

E-mail: zlf0101@aliyun.com

Key words: chondroitin sulfate, $\mathrm{CHSY} 1, \mathrm{NF \kappa B}$, caspase-3/7, RKO cell line in colorectal cancer. The present study also suggests that CHSY1 may be a potential target for colorectal cancer therapy.

\section{Introduction}

Chondroitin sulfate (CS) is a class of glycosaminoglycan (GAG), which is mainly present in the extracellular matrix and on the cell surface (1). CS plays very important roles in morphogenesis and tissue development (2). CS also shows an immunomodulatory effect and has been reported to be involved in tumor progression $(3,4)$. For example, in colorectal cancer, the GAG disaccharide content and composition were altered (5). Additionally, in endometrial epithelial cancer, CS promoted cell proliferation and migration (3). Chondroitin synthase-1 (CHSY1) is one of the six enzymes responsible for the biosynthesis of CS in mammalian cells (6). CHSY1is a protein with 802 amino acids and is located in the chromosome $15 \mathrm{q} 26.3$ region. CHSY1 is important for normal development. For example, the methylation level of CHSY1 is associated with T cell differentiation (7). CHSY1 is also necessary for bone development (8), and loss of CHSY1 causes temtamy preaxial brachydactyly syndrome (9).

However, evidence suggests an oncogenic function of CHSY1 during tumorigenesis. For example, CHSY1 is required for the interaction of myeloma cells with osteoclasts (10). The abnormal expression of CHSY1 has been found in malignant soft tissue sarcomas (11). Furthermore, knockdown of CHSY1 increased the expression of JAG2, a critical molecule in glioblastoma cells (12). Forced expression of CHSY1 enhanced cell migration, invasion, and EMT in hepatocellular carcinoma (13). As a result, CHSY1 was proposed to promote tumor progression. However, in colorectal cancer, CHSY1 expression showed a significant increase in stage I tumor tissues compared to that in the normal control group. In stage II or III tumor tissues, expression of CHSY1 was comparable or slightly lower than that in control tissues (14). However, the actual function of CHSY1 in colorectal cancer remains unknown.

According to cancer reports by Chen, colorectal cancer is one of the most four malignant cancers in China. The estimated number of new cases was 376,300 and the number of new deaths was $\sim 191,000$ (15). Colorectal cancer is also a 
common malignant cancer in the USA. As reported, both the new incidence and new mortality of colorectal cancer patients accounted for $\sim 8 \%$ of all cancers in 2017 (16). Furthermore, the 5-year survival rate of metastatic colorectal cancer patients was $<15 \%$ (17). However, surgical resection remains the most commonly used therapy for colorectal cancer (17). Unfortunately, $\sim 50 \%$ of colorectal cancer patients undergo recurrence and metastasis following surgery (18). Therefore, it is necessary to determine the mechanisms underlying colorectal cancer and develop new strategies to win the war against colorectal cancer.

In this study, to investigate the role of CHSY1 in colorectal cancer, we determined the clinical level of CHSY1 in tumor tissues and evaluated the effects of CHSY1 on cell growth and cell apoptosis. Then, we demonstrated that nuclear factor (NF) $\kappa \mathrm{B}$ and caspase-3/7 signaling were regulated by CHSY1.

\section{Materials and methods}

Cell lines and cell culture. Human colorectal cancer cell lines, including RKO, HCT116, SW480 and the human immortal colon epithelial cell line NCM460, were obtained from the Shanghai Cell Bank of Chinese Academy of Science (Shanghai, China) and maintained in RPMI-1640 medium (Gibco; Thermo Fisher Scientific, Inc., Waltham, MA, USA) supplemented with $10 \%$ fetal bovine serum (Sangon Biotech, Shanghai, China), 100 units $/ \mathrm{ml}$ penicillin and $0.1 \mathrm{mg} / \mathrm{ml}$ streptomycin at $37^{\circ} \mathrm{C}$ in a $5 \% \mathrm{CO}_{2}$ incubator.

Patient tissues and ethics statement. A total of 21 tumor tissues and the adjacent normal tissues were collected from Jiangxi Province People's Hospital (Nanchang, China) between 2009 and 2012.

All study procedures were approved by the Institutional Review Board of Jiangxi Provincial People's Hospital, and a written informed consent form was collected from each patient.

Immunohistochemistryassay (IHC). The IHC assay was carried out as report before (19). Briefly, tissue sections of $4 \mu \mathrm{m}$ were deparaffinized, rehydrated, and subjected to antigen retrieval by boiling in sodium citrate buffer $(10 \mathrm{mmol} / \mathrm{l}$; $\mathrm{pH}$ 6.0). Then the sections were incubated with CHSY1 primary antibody (ab153813; 1:400 dilution; Abcam, Cambridge, MA, USA) for $60 \mathrm{~min}$ at room temperature and stained with 3,3-diaminobenzidine followed by counterstaining with hematoxylin and mounted. The stains were scored according to: (a) percentage of immune-positive cells, 1, 0-30\%; 2, >30-70\%; 3, >70\%; and (b) staining intensity, 1 , weak; 2 , moderate and 3 , strong. The final score of each slide was (a) $\mathrm{x}$ (b).

Reverse transcription-quantitative polymerase chain reaction $(R T-q P C R)$. Total RNA was extracted using TRIzol reagent (Invitrogen; Thermo Fisher Scientific, Inc.) and cDNA was synthesized with a PrimeScript First Strand cDNA Synthesis kit (Takara, Dalian, China) according to the manufacturer's instructions. Next, $1 \mu \mathrm{l}$ of cDNA was used as a template for the RT-qPCR assay with SYBR Green reagent on a 7500 Fast Real-Time PCR System (Applied Biosystems, Foster City, CA, USA). The primers designed for CHSY1 gene were as follows:
Forward, 5'-GCTATCACATTACACCCCAACA-3' and reverse, 5'-AACTCCCATTCCAGAATCTCCT-3'

GAPDH was selected as an internal control and the primers were as follows: Forward, 5'-TGACTTCAACAGCGACAC CCA-3' and reverse, 5'-CACCCTGTTGCTGTAGCCAAA-3'

The protocol for RT-qPCR was as follows: Denaturation at $95^{\circ} \mathrm{C}$ for $20 \mathrm{sec}$, (denaturation at $95^{\circ} \mathrm{C}$ for $5 \mathrm{sec}$, extension at $60^{\circ} \mathrm{C}$ for $30 \mathrm{sec}$ ) for 40 cycles.

The expected PCR products of CHSY1 and GAPDH were 236 and $121 \mathrm{bp}$, respectively. All samples were examined in triplicate. The relative level of the target gene was calculated using the $2^{-\Delta \Delta \mathrm{Cq}}$ as described previously (20). The expression level of CHSY1 was considered as high when the fold change of CHSY1 in tumor tissues vs. that in normal control was $>2$. Otherwise, it was considered as low.

Construction of recombinant lentiviral vector and transduction. The shRNA fragment targeting human the CHSY1 gene (GenBank no. NM_014918) was designed, synthesized, and inserted into a lentivirus expression plasmid pGV115-GFP. The shRNA sequence was as follows: 5-ACATTGTCATGCAGG TCAT-3. Then, the lentivirus particle carrying this shRNA fragment (shCHSY1) was prepared.

After the lentivirus particle was prepared, approximately $2 \times 10^{5} \mathrm{RKO}$ cells/well were cultured in 6-well plates and infected with shCHSY1 lentivirus or control lentivirus (shCtrl) at a multiplicity of infection (MOI) of 20. Then, the treated cells were incubated in a $5 \% \mathrm{CO}_{2}$ incubator at $37^{\circ} \mathrm{C}$ for 5 days. After $72 \mathrm{~h}$ of infection, cells were observed under a fluorescence microscope (MicroPublisher 3.3RTV; Olympus, Tokyo, Japan). After 5 days of infection, the knockdown efficiency of CHSY1 was determined using RT-qPCR and western blotting technologies.

Cell proliferation assay. Cell growth viability was monitored on a Cellomics ArrayScan ${ }^{\mathrm{TM}}$ VT1 HCS automated reader (Cellomics Inc., Pittsburgh, PA, USA). Briefly, RKO cells infected with lentivirus were seeded into 96 -well plates $\left(2,000\right.$ cells/well) and incubated for 5 days at $37^{\circ} \mathrm{C}$ in a $5 \% \mathrm{CO}_{2}$ incubator, and the cell number was calculated each day for 5 days according to the GFP expression intensity. Each experiment was performed in triplicate.

MTT assay. SW480 cells or RKO cells treated with shCHSY1 lentivirus or shCtrl were seeded into 96 -well plates at 6,000 cells/well and cultured for $48 \mathrm{~h}$ at $37^{\circ} \mathrm{C}$ in a $5 \% \mathrm{CO}_{2}$ incubator. Then MTT reagent $(5 \mathrm{mg} / \mathrm{ml}$; Sangon Biotech) was added into each well and cultured for another $4 \mathrm{~h}$. The absorbance value at $490 \mathrm{~nm}$ was detected on a microplate spectrophotometer.

Apoptosis analysis. The cell apoptosis rate was determined with Annexin V-APC staining by flow cytometry. Briefly, RKO cells (5,000 cells/well) were cultured in 6-well plates. After $48 \mathrm{~h}$ of lentivirus infection, cells were collected and washed twice with ice-cold PBS. Then, cells were adjusted to $1 \times 10^{6} / \mathrm{ml}$ with $1 \mathrm{X}$ staining buffer (Sangon Biotech), of which $100 \mu 1$ of the cell suspension was stained with $5 \mu 1$ Annexin V-APC (BD Biosciences, San Diego, CA, USA) for $15 \mathrm{~min}$ at room temperature in the dark. Then, the cells were analyzed 

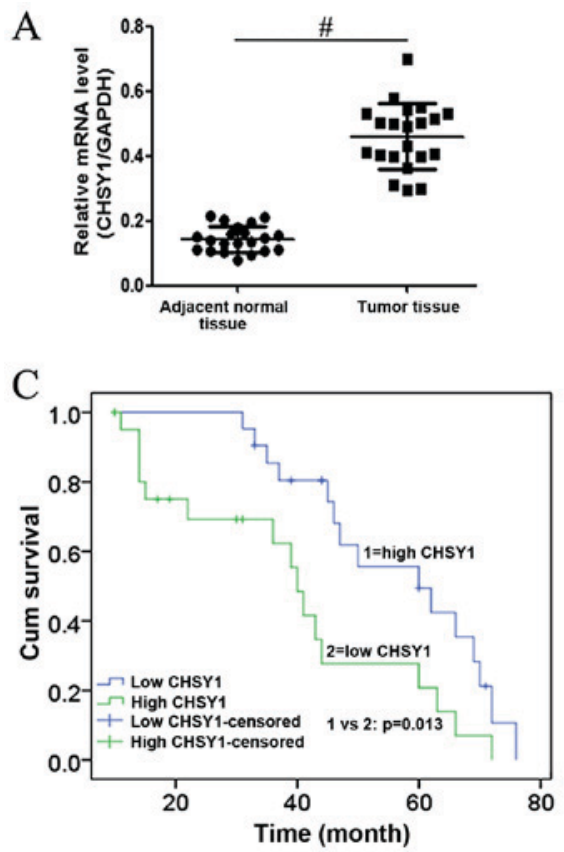

B

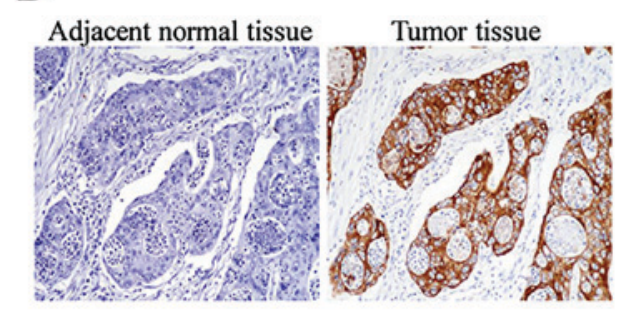

D

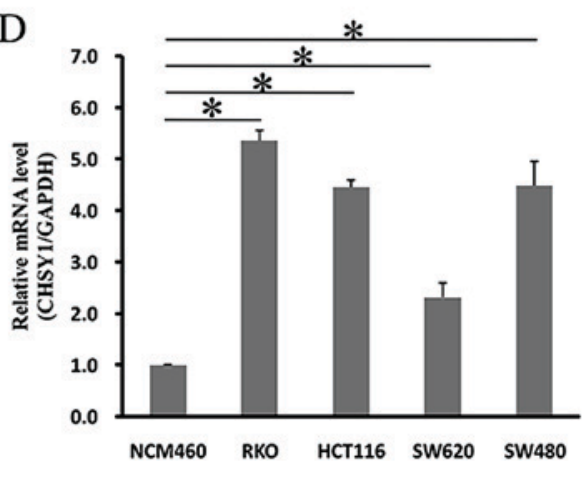

Figure 1. CHSY1 was overexpressed in colorectal cancer and associated with prognosis. (A) The expression of CHSY1 was much higher in 21 colorectal cancer tissues than in the adjacent normal tissues. (B) Immunohistochemistry analysis of CHSY1 in tumor or adjacent normal tissues (magnification, $\mathrm{x} 40$ ). (C) The 5-year survival rate of patients with a high level of CHSY1 was worse than those with low CHSY1 expression. (D) RT-qPCR analysis showed that CHSY1 was highly expressed in colorectal cancer cell lines. ${ }^{\#} \mathrm{P}<0.05$ indicated significant difference of tumor tissues vs. adjacent normal tissues. "P $<0.05$ indicated significance of tumor cell lines vs. NCM460. CHSY1, chondroitin synthase-1; RT-qPCR, reverse transcription-quantitative polymerase chain reaction.

on a flow cytometer. Each experiment was performed independently three times.

Caspase-3/7 activity assay. To detect the activity of caspase-3/7, we seeded the RKO cells into 96-well plates and infected them with the lentivirus as described above. After infection for $48 \mathrm{~h}$, the activity of caspase-3/7 was determined with a Caspase-Glo 3/7 kit (Promega Corp., Madison, WI, USA) according to the manufacturer's instructions.

Western blot analysis. After $48 \mathrm{~h}$ of lentivirus infection, approximately $1 \times 10^{6}$ cells were collected and lysed with lysis buffer (50 mM Tris, pH 7.4, 150 mM NaCl, 1\% SDS, 1 mM EDTA, and $1 \%$ NP-40) containing $1 \mathrm{mM}$ PMSF (Sangon Biotech) for $30 \mathrm{~min}$ on ice. Then, the lysates were centrifuged at $10,000 \mathrm{x} \mathrm{g}$ for $10 \mathrm{~min}$ at $4^{\circ} \mathrm{C}$, and the supernatants were collected. The protein concentration was determined using a BCA Protein Assay kit (Generay, Shanghai, China). Then, approximately $10 \mu \mathrm{g}$ of protein was separated on a $10 \%$ SDS-PAGE gel and transferred to a polyvinylidenedifluoride (PVDF) membrane.

PVDF membranes were incubated with mouse anti-CHSY1 (1:200 dilution; ab153813), anti-NFкB p105/p50 (1:300 dilution; ab131546), anti-B-cell lymphoma 2 (Bcl-2; 1:500 dilution; ab32124), anti-truncated caspase-3/7 (1:1,000 dilution; ab2302), anti-Bcl-2-associated X protein (Bax; 1:400 dilution; ab182733; Abcam), anti-Pi-IкB (1:200 dilution; sc8404), or anti-GAPDH antibody (1:1,500 dilution; sc47724; Santa Cruz Biotechnology, Santa Cruz, CA, USA) at $4^{\circ} \mathrm{C}$ overnight. Then, the PVDF membranes were subsequently incubated with a horseradish peroxidase (HRP)-conjugated goat anti-mouse IgG (1:1,500 dilution; sc2005) or goat anti-rabbit $\operatorname{IgG}(1: 1,200$ dilution; sc2030; Santa Cruz Biotechnology) at $37^{\circ} \mathrm{C}$ for $1 \mathrm{~h}$ and detected with the EasyBlot ECL kit (Sangon Biotech).
Statistical evaluation. For in vitro experiments, statistical analyses were performed with SPSS 16.0 (SPSS, Chicago, IL, USA). Data are expressed as the mean \pm SD. Raw data was subjected to Independent Samples t-test to analyze the difference between group shCtrl and shCHSY1. The difference between multiple groups was analyzed by one-way ANOVA/post hoc Tukey Test. The difference of CHSY1 expression between tumor tissues and adjacent normal tissues was analyzed with paired Student's t-test. $\mathrm{P}<0.05$ was considered to indicate a statistically significant difference.

For the association analysis of CHSY1 expression with the prognosis of patients, the Kaplan-Meier method was used, and a log-rank test was employed to analyze the difference. $\mathrm{P}<0.05$ was considered to indicate a statistically significant difference.

\section{Results}

CHSY1 is clinically associated with colorectal cancer. To explore the relationship of CHSY1 with colorectal cancer, we collected a total of 21 tumor samples and adjacent normal samples. Then, the level of CHSY1 was detected using the RT-qPCR method. As shown in Fig. 1A, CHSY1 was more highly expressed in tumor samples than in adjacent normal samples. IHC staining also demonstrated that CHSY1 was expressed highly in tumor tissues and was weak in the adjacent normal control (Fig. 1B and Table I). Furthermore, higher CHSY1 expression was associated with a poorer prognosis, such that the 5-year survival rate of patients with high CHSY1 (tumor: Normal $\geq 2$ ) was significantly lower than those with low CHSY1 expression (tumor: Normal <2) (20\% vs. 45\%) (Fig. 1C). In addition, we found that CHSY1 was more highly expressed in colorectal cancer cell lines, including RKO, HCT116, and SW480, than in NCM460 cells, 
Table I. Mean score of CHSY1 expression in tumor tissues or adjacent normal tissues by IHC analysis.

\begin{tabular}{lccc} 
Group & Adjacent normal tissues & Tumor tissues & P-value \\
\hline Score value & $0.52 \pm 0.51$ & $4.24 \pm 2.43$ & $<0.05^{\mathrm{a}}$
\end{tabular}

aSignificant difference between tumor tissues and adjacent normal tissues. CHSY1, chondroitin synthase-1; IHC, immunohistochemistry.

a human colon epithelial cell (Fig. 1D). Therefore, CHSY1 was clinically associated with colorectal cancer.

CHSY1 was successfully knocked down by lentivirus-mediated shRNA in colorectal cancer cells. The lentivirus vector was an efficient tool to carry a particular gene into cells and was applied extensively. To reduce the expression of CHSY1 in the RKO cell line, we synthesized a shRNA fragment targeting CHSY1 (shCHSY1) and the negative control (shCtrl) and prepared lentivirus particles carrying shCHSY1 or shCtrl. Because GFP was a tag in the lentivirus vector, the infection efficiency of shCHSY1 in RKO cells could be monitored directly under a microscope. As shown in Fig. 2A, the prepared lentivirus particles efficiently infected RKO cells. And CHSY1 expression was significantly reduced in RKO cells at protein level (Fig. 2B). Also, the mRNA level of CHSY1 was decreased greatly by shCHSY1 in RKO cells. The knockdown efficiency was $\sim 70 \%$. Therefore, CHSY1 was successfully knocked down in the RKO cell line.

CHSY1 serves critical roles in the proliferation of colorectal cancer cells. To examine the effects of CHSY1 knockdown on cell proliferation, we treated RKO cells seeded in a 96-well plate with shCHSY1 or shCtrl and monitored the cells for 5 consecutive days with Cellomics. As shown in Fig. 3A, the GFP intensity in the shCtrl group was higher than that in the shCHSY1 group, which indicated that RKO cells treated with shCtrl underwent significant expansion after culture for 5 days. This was further supported by Fig. 3B and D. The cell number in the shCtrl group doubled compared to that in the shCHSY1 group. In addition, CHSY1 was effectively reduced in SW480 cells (Fig. 3C), and the proliferation of SW480 cells was inhibited after CHSY1 knockdown (Fig. 3D). Therefore, we hypothesized that CHSY1 was essential for cell proliferation in colorectal cancer.

Knockdown of CHSY1 induces apoptosis in RKO cells. In tumor cells, apoptosis was often suppressed by a driver gene. Not surprisingly, we found that decreased expression of CHSY1 increased cell apoptosis in RKO cells. As demonstrated in Fig. 4A and B, the apoptosis rate of cells treated with shCtrl was $5.09 \%$, whereas it was $14.15 \%$ in the shCHSY1 group. The difference between the two groups was significant $(\mathrm{P}<0.05)$. Then, the activity of caspase- $3 / 7$ was determined in RKO cells, and the activity of caspase- $3 / 7$ in the shCHSY1 group was approximately 6-foldthat in the shCtrl group (Fig. 4C). The above data suggested that CHSY1 played important roles in the apoptosis of RKO cells.

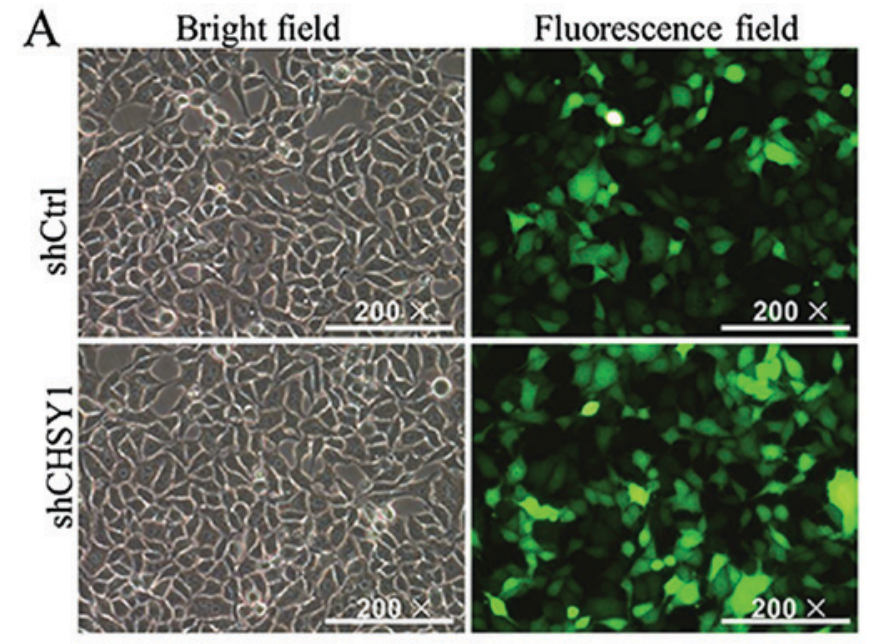

B

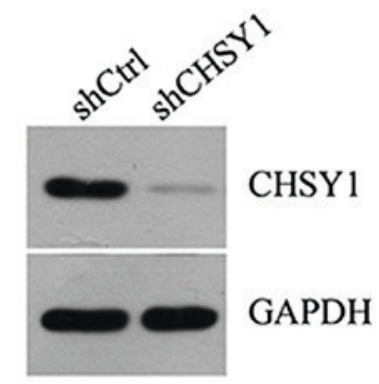

Figure 2. CHSY1 was successfully knocked down in RKO cells. (A) The expression of GFP demonstrated that the lentivirus vector carrying shCtrl or shCHSY1 was efficiently transduced into RKO cells. (B) Western blotting analysis showed that CHSY1 expression was greatly reduced in RKO cells at the protein level. CHSY1, chondroitin synthase-1; Ctrl, control.

CHSY1 regulates $N F \kappa B$ and caspase-3/7 signaling in RKO cells. To elucidate the mechanism by which CHSY1 affects cell proliferation and apoptosis in colorectal cancer, we determined the critical signaling molecules in proliferation and apoptosis. As shown in Fig. 5, expression of the anti-apoptotic molecule Bcl-2 was decreased after CHSY1 was knocked down in RKO cells. In contrast, the level of the pro-apoptotic molecule Bax increased significantly, and the level of truncated caspase-3/7 was also increased. Additionally, we found that the phos-

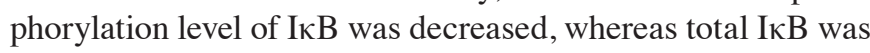
increased after CHSY1 knockdown. Moreover, the expression of NFKB was also reduced. Conversely, overexpression of CHSY1 increased the level of NFKB and the phosphorylated level of IкB; however, total ІкB expression was decreased. Moreover, Bcl-2 was upregulated, whereas Bax and truncated caspase-3/7 levels were reduced. Therefore, we hypothesized that CHSY1 regulated cell proliferation and apoptosis via regulation of the $\mathrm{NF \kappa B}$ and/or caspase-3/7 signaling pathway in RKO cells.

\section{Discussion}

Colorectal cancer is one of the most common malignant diseases in the world and threatens the life of humans. The most effective weapon against colorectal cancer is just a scalpel, but we are currently losing the war. One of the major causes of the poor prognosis in colorectal cancer is the heterogeneity of the cancer (21). Although a number of factors have 


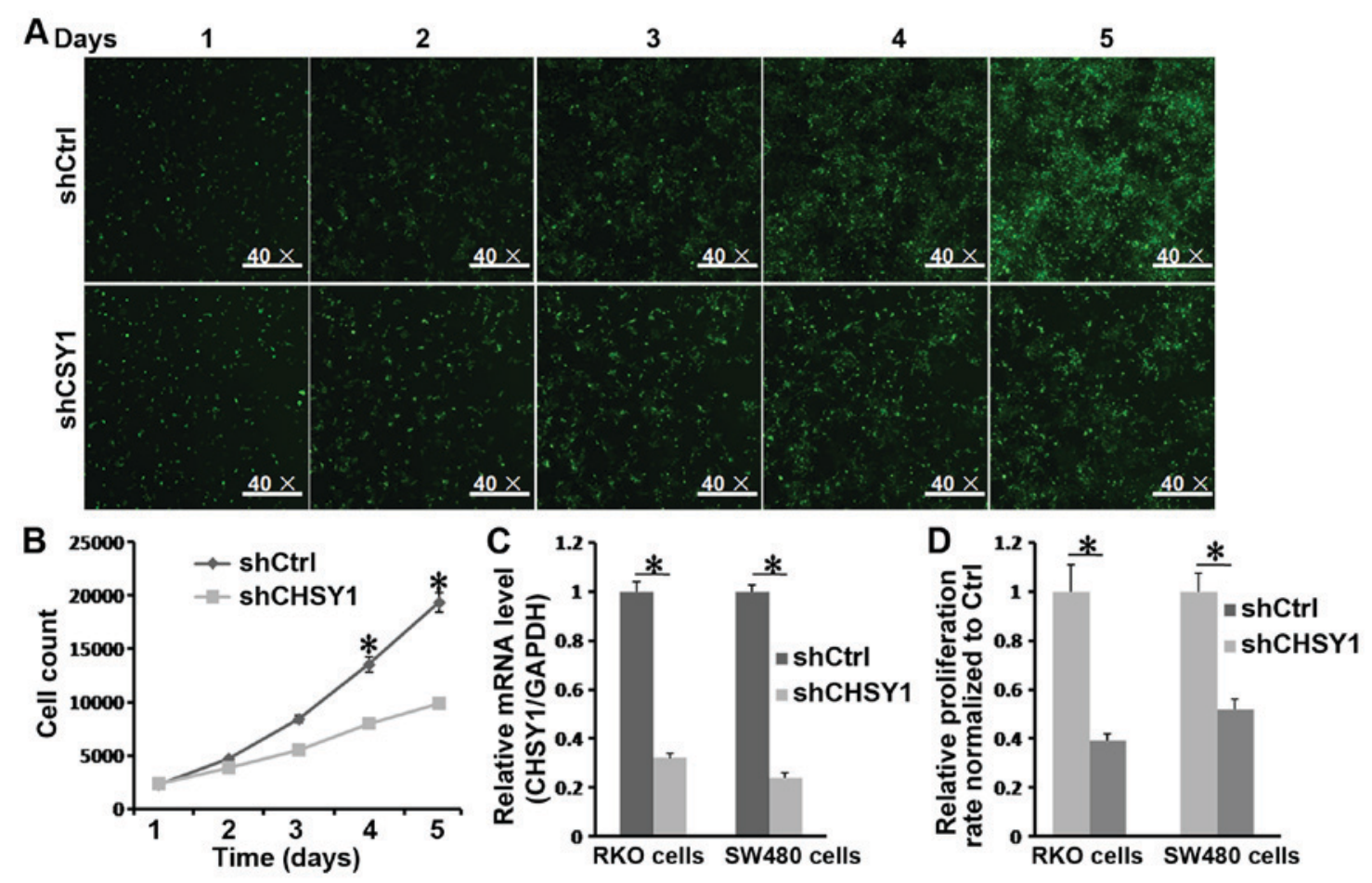

Figure 3. CHSY1 knockdown inhibited the proliferation of RKO and SW480 cells. (A) The growth of RKO cells was consecutively monitored for 5 days under fluorescence imaging. (B) The number of cells was counted each day for 5 days. (C) RT-qPCR analysis showed that CHSY1 was knocked down in SW480 and RKO cells. (D) MTT assay demonstrated that CHSY1 knockdown inhibited the proliferation of SW480 and RKO cells. "P $<0.05$ indicated a significant difference between group shCHSY1 and shCtrl. CHSY1, chondroitin synthase-1; Ctrl, control; RT-qPCR, reverse transcription-quantitative polymerase chain reaction.
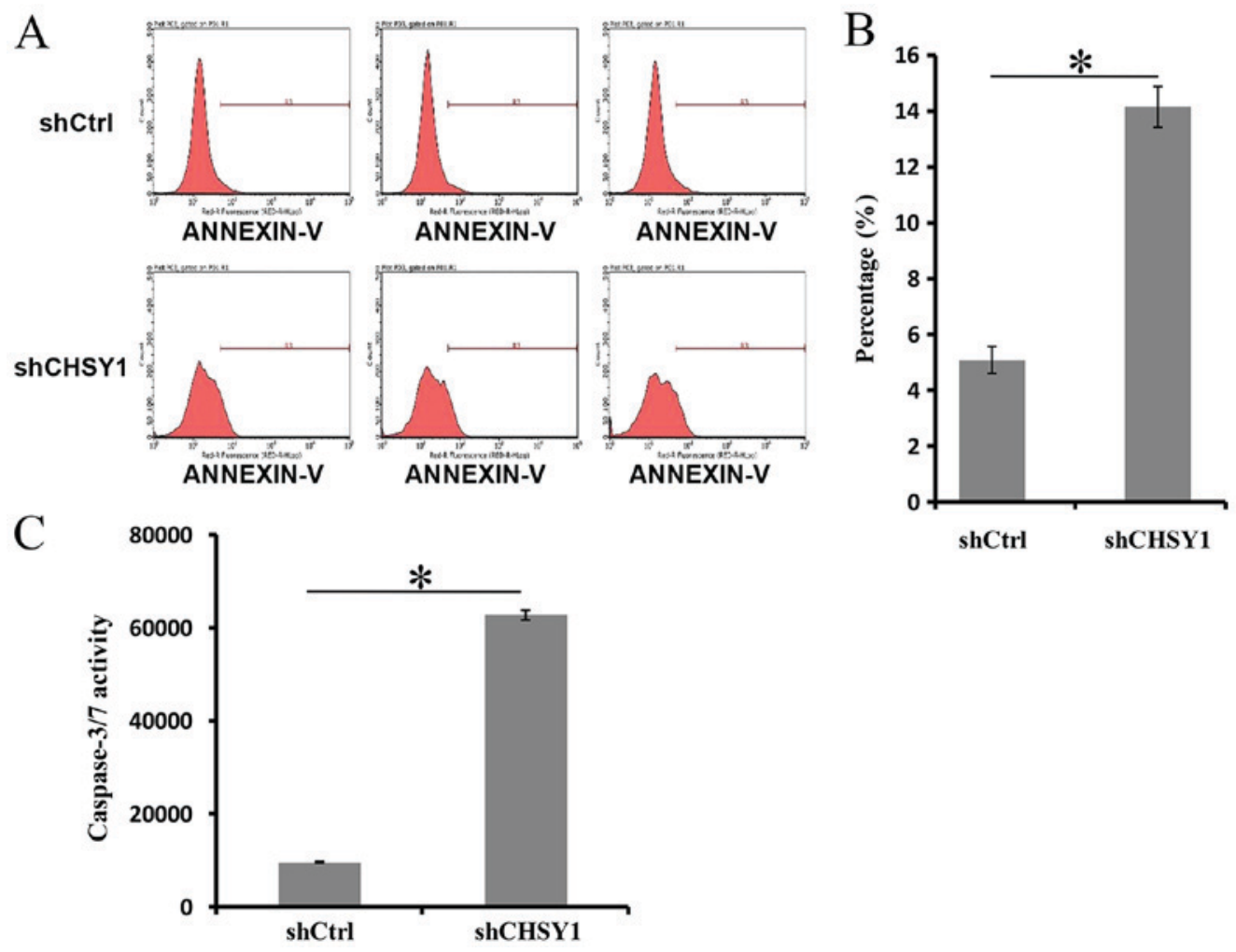

Figure 4. CHSY1 knockdown induced apoptosis in RKO cells. (A) The flow cytometric analysis of RKO cells cultured for $48 \mathrm{~h}$ after lentivirus infection. (B) The percentage of apoptotic cells after RKO cells were infected with shCHSY1 or shCtrl lentivirus. (C) The activity of caspase-3/7 was determined with a caspase-Glo 3/7 kit. "P<0.05 indicated a significant difference between group shCHSY1 and shCtrl. CHSY1, chondroitin synthase-1; Ctrl, control.

been identified, none are able to fully explain the formation or progression of cancer. In this study, we demonstrated that
CHSY1 was expressed more highly in colorectal cancer tissues and colorectal cancer cell lines than in control tissues and 
A

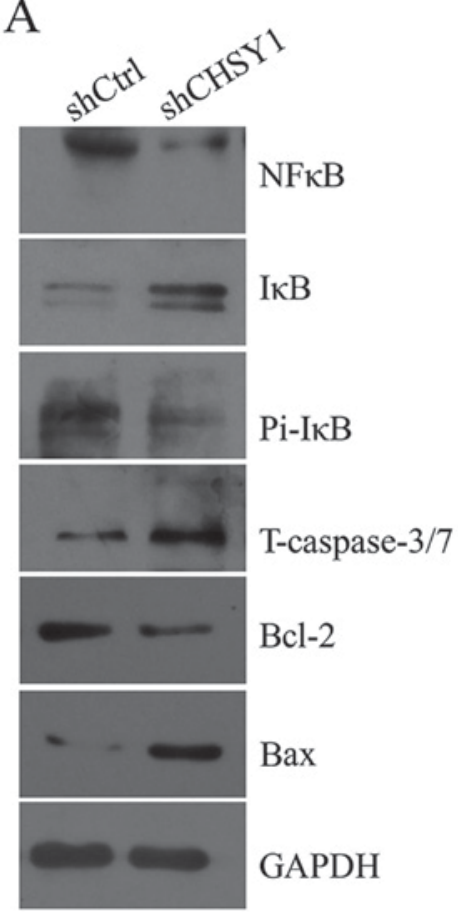

B

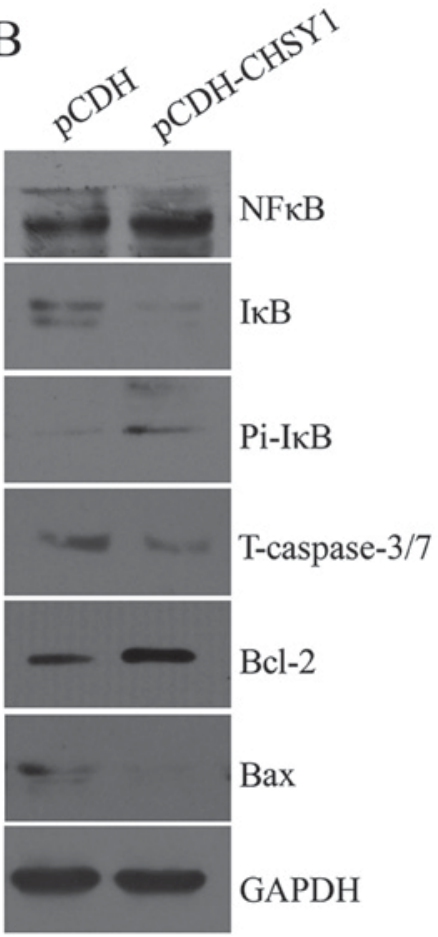

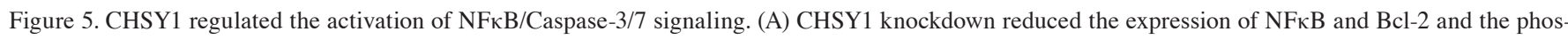

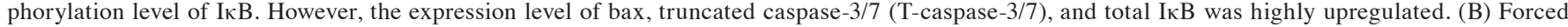

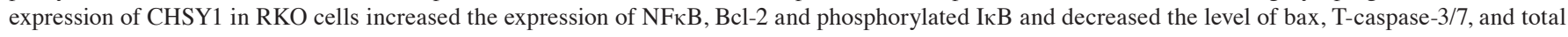
I $\mathrm{B}$. NF, nuclear factor; CHSY1, chondroitin synthase-1; Bcl, B-cell lymphoma.

lines. Moreover, higher CHSY1 expression was associated with a worse 5-year survival rate. These data indicate that CHSY1 may play critical roles in the progression of colorectal cancer. In a previous study, CHSY1 was significantly upregulated in stage I colorectal cancer but not in stage II or III (14). In this study, we showed that CHSY1 was more highly expressed in both stage II and III colorectal cancer tissues than in the adjacent normal control. The difference may be partially attributed to the origin of the tumor tissues. However, a large cohort of patient tissues was necessary to clearly explain this paradox and to confirm the discovery in this study.

Nearly all types of cancers are characterized by rapid expansion and evasion from drug-induced apoptosis (22). Here, we showed that CHSY1 was essential for the proliferation of colorectal cancer cell lines, including RKO cells and SW480 cells. The NFkB signaling pathway was often activated in tumors and promoted cell proliferation $(23,24)$. In RKO cells, the level of $\mathrm{NF} \kappa \mathrm{B}$ and phosphorylated $\mathrm{I} \kappa \mathrm{B}$ was decreased, whereas total $\mathrm{I} \kappa \mathrm{B}$ was increased after CHSY1 knockdown. Conversely, CHSY1 overexpression increased the level of NFאB and phosphorylated $\mathrm{I} \kappa \mathrm{B}$ in RKO cells. In tumors, I $\mathrm{R}$ B was phosphorylated and separated from the NFKB molecule. When

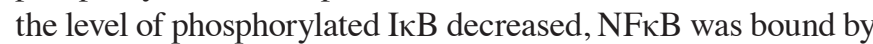
$\mathrm{I} \kappa \mathrm{B}$, and the expression of downstream genes was inhibited (25). Therefore, CHSY1 suppressed cell proliferation via regulation of the NFאB signaling pathway in colorectal cancer.

Additionally, in RKO cells, CHSY1 could suppress cell apoptosis, which may partially account for the poor prognosis of colorectal cancer patients. The major mechanism of a large number of chemotherapeutic drugs in the clinic is apoptosis $(26,27)$. Generally, chemotherapeutic drugs further clear remaining cancer cells by inducing cell apoptosis after the surgical removal of solid tumor tissues, and patients do benefit from this treatment. However, a small cohort of cancer cells is able to evolve and evade the apoptosis induced by drugs.

Apoptosis is a programmed cell death process, and the caspase cascade response is active when apoptosis begins. In general, the activity of caspase-3/7 is greatly enhanced in the apoptosis process $(28,29)$. In this study, CHSY1 was shown to contribute to the anti-apoptotic ability of RKO cells, and caspase-3/7 was highly activated after CHSY1 was knocked down in RKO cells. Furthermore, we found that Bcl-2 was decreased, whereas Bax increased after knockdown of CHSY1. Bcl-2 is an antagonistic gene of apoptosis (28), but Bax often promotes the progression of apoptosis $(28,29)$. The expression pattern suggested that caspase-3/7-mediated apoptosis signaling was activated when CHSY1 was knocked down in RKO cells.

However, these results were just based on RKO cells. And it is necessary to confirm the role of CHSY1 in another cell line such as SW480 cells. Also, the in vivo function of CHSY1 gene in colorectal cancer will be explored and the dominant molecular mechanism of CHSY1 in colorectal cancer will be an emphasis in future.

In summary, we demonstrated that CHSY1 played a tumor-promoting role in colorectal cancer by regulating the $\mathrm{NF} \kappa \mathrm{B}$ and/or caspase-3/7 signaling pathway. Additionally, this study suggests that CHSY1 is a potential target for colorectal cancer therapy.

\section{Acknowledgements}

Not applicable. 


\section{Funding}

No funding was received.

\section{Availability of data and materials}

The datasets used and/or analyzed during the current study are available from the corresponding author on reasonable request.

\section{Authors' contributions}

LZ designed the whole study, carried out the experiments and wrote the manuscript. JQ analyzed the data. XL contributed to the acquisition of data, carried out the western blot experiments, participated in the draft and revision of manuscript and studied the references.. AZ and $\mathrm{ZZ}$ participated in the experiments and interpreted the immunohistochemistry data. QF reviewed the manuscript and contributed to the acquisition of data.

\section{Ethics approval and consent to participate}

All study procedures were approved by the Institutional Review Board of Jiangxi Provincial People's Hospital, and a written informed consent form was collected from each patient.

\section{Patient consent for pubication}

The patients provided written informed consent for the publication of any associated data.

\section{Competing interests}

The authors declare that they have no competing interests.

\section{References}

1. Martel-Pelletier J, Boileau C, Pelletier JP and Roughley PJ: Cartilage in normal and osteoarthritis conditions. Best Pract Res Clin Rheumatol 22: 351-384, 2008.

2. Holmborn K, Habicher J, Kasza Z, Eriksson AS, Filipek-Gorniok B, Gopal S, Couchman JR, Ahlberg PE, Wiweger M, Spillmann D, et al: On the roles and regulation of chondroitin sulfate and heparin sulfate in zebrafish pharyngeal cartilage morphogenesis. J Biol Chem 287: 33905-33916, 2012.

3. Winship A, Van Sinderen M, Heffernan-Marks A and Dimitriadis E: Chondroitin sulfate proteoglycan protein is stimulated by interleukin 11 and promotes endometrial epithelial cancer cell proliferation and migration. Int J Oncol 50: 798-804, 2017.

4. du Souich P, García AG, Vergés J and Montell E: Immunomodulatory and anti-inflammatory effects of chondroitin sulphate. J Cell Mol Med 13: 1451-1463, 2009.

5. Kalathas D, Theocharis DA, Bounias D, Kyriakopoulou D, Papageorgakopoulou N, Stavropoulos MS and Vynios DH: Alterations of glycosaminoglycan disaccharide content and composition in colorectal cancer: Structural and expressional studies. Oncol Rep 22: 369-375, 2009.

6. Ogawa H, Hatano S, Sugiura N, Nagai N, Sato T, Shimizu K, Kimata K, Narimatsu $\mathrm{H}$ and Watanabe $\mathrm{H}$ : Chondroitin sulfate synthase-2 is necessary for chain extension of chondroitin sulfate but not critical for skeletal development. PLoS One 7: e43806, 2012.

7. Hashimoto SI, Ogoshi K, Sasaki A, Abe J, Qu W, Nakatani Y, Ahsan B, Oshima K, Shand FH, Ametani A, et al: Coordinated changes in DNA methylation in antigen-specific memory CD4 T cells. J Immunol 190: 4076-4091, 2013.

8. Wilson DG, Phamluong K, Lin WY, Barck K, Carano RA, Diehl L, Peterson AS, Martin F and Solloway MJ: Chondroitin sulfate synthase 1 (Chsy1) is required for bone development and digit patterning. Dev Biol 363: 413-425, 2012.
9. Li Y, Laue K, Temtamy S, Aglan M, Kotan LD, Yigit G, Canan H, Pawlik B, Nürnberg G, Wakeling EL, et al: Temtamy preaxial brachydactyly syndrome is caused by loss-of-function mutations in chondroitin synthase 1, a potential target of BMP signaling. Am J Hum Genet 87: 757-767, 2010.

10. Yin L: Chondroitin synthase 1 is a key molecule in myeloma cell-osteoclast interactions. J Biol Chem 280: 15666-15672, 2005.

11. Momose T, Yoshimura Y, Harumiya S, Isobe K, Kito M, Fukushima M, Kato $\mathrm{H}$ and Nakayama J: Chondroitin sulfate synthase 1 expression is associated with malignant potential of soft tissue sarcomas with myxoid substance. Hum Pathol 50: $15-23,2016$.

12. Tian J, Ling L, Shboul M, Lee H, O'Connor B, Merriman B, Nelson SF, Cool S, Ababneh OH, Al-Hadidy A, et al: Loss of CHSY1, a secreted FRINGE enzyme, causes syndromic brachydactyly in humans via increased NOTCH signaling. Am J Hum Genet 87: 768-778, 2010.

13. Liu CH, Lan CT, Chou JF, Tseng TJ and Liao WC: CHSY1 promotes aggressive phenotypes of hepatocellular carcinoma cells via activation of the hedgehog signaling pathway. Cancer Lett 403: 280-288, 2017.

14. Kalathas D, Theocharis DA, Bounias D, Kyriakopoulou D, Papageorgakopoulou N, Stavropoulos MS and Vynios DH: Chondroitin synthases I, II, III and chondroitin sulfate glucuronyltransferase expression in colorectal cancer. Mol Med Rep 4: 363-368, 2011.

15. Chen W, Zheng R, Baade PD, Zhang S, Zeng H, Bray F, Jemal A, $\mathrm{Yu}$ XQ and He J: Cancer statistics in China, 2015. CA Cancer J Clin 66: 115-132, 2016.

16. Siegel RL, Miller KD and Jemal A: Cancer statistics, 2017. CA Cancer J Clin 67: 7-30, 2017.

17. Pabla B, Bissonnette M and Konda VJ: Colon cancer and the epidermal growth factor receptor: Current treatment paradigms, the importance of diet, and the role of chemoprevention. World J Clin Oncol 6: 133-141, 2015.

18. Sauer R, Liersch T, Merkel S, Fietkau R, Hohenberger W, Hess C, Becker H, Raab HR, Villanueva MT, Witzigmann H, et al: Preoperative versus postoperative chemoradiotherapy for locally advanced rectal cancer: Results of the German CAO/ARO/AIO-94 randomized phase III trial after a median follow-up of 11 years. J Clin Oncol 30: 1926-1933, 2012.

19. Luo Y, Tsuchiya KD, II Park D, Fausel R, Kanngurn S, Welcsh P, Dzieciatkowski S, Wang J and Grady WM: RET is a potential tumor suppressor gene in colorectal cancer. Oncogene 32: 2037-2047, 2013.

20. Livak KJ and Schmittgen TD: Analysis of relative gene expression data using real-time quantitative PCR and the 2(-Delta Delta C(T)) method. Methods 25: 402-408, 2001.

21. Fisher R, Pusztai L and Swanton C: Cancer heterogeneity: Implications for targeted therapeutics. $\mathrm{Br} \mathrm{J}$ Cancer 108: 479-485, 2013.

22. Hanahan D and Weinberg RA: Hallmarks of cancer: The next generation. Cell 144: 646-674, 2011.

23. Xia JT, Chen LZ, Jian WH, Wang KB, Yang YZ, He WL, He YL, Chen D and Li W: MicroRNA-362 induces cell proliferation and apoptosis resistance in gastric cancer by activation of $\mathrm{NF}-\kappa \mathrm{B}$ signaling. J Transl Med 12: 33, 2014.

24. Cui H, Yuan J, Du X, Wang M, Yue L and Liu J: Ethyl gallate suppresses proliferation and invasion in human breast cancer cells via Akt-NF- $\kappa$ B signaling. Oncol Rep 33: 1284-1290, 2015.

25. Li F, Zhang J, Arfuso F, Chinnathambi A, Zayed ME, Alharbi SA, Kumar AP, Ahn KS and Sethi G: NF-кB in cancer therapy. Arch Toxicol 89: 711-731, 2015.

26. Makin G and Hickman JA: Apoptosis and cancer chemotherapy. Cell Tissue Res 301: 143-152, 2000.

27. Dandekar DS, Lopez M, Carey RI and Lokeshwar BL: Cyclooxygenase-2 inhibitor celecoxib augments chemotherapeutic drug-induced apoptosis by enhancing activation of caspase-3 and -9 in prostate cancer cells. Int J Cancer 115: 484-492, 2005.

28. Wong RS: Apoptosis in cancer: From pathogenesis to treatment. J Exp Clin Cancer Res 30: 87, 2011.

29. Vermeulen K, Van Bockstaele DR and Berneman ZN: Apoptosis: Mechanisms and relevance in cancer. Ann Hematol 84: 627-639, 2005. 